\section{Perioperative Management of Groin Ascitic Hernia by Hernia-roof Technique: A 14-year Experience}

\section{Abstract}

Objective: To describe and evaluate the clinical value of preperitoneal tensionfree repair of inguinal hernias combined with ascites via the hernia sac top (HST) pathway.

Methods: We retrospectively reviewed the medical records of seven patients with inguinal hernia combined with ascites who underwent preperitoneal tension-free repair via the HST pathway from January 2010 to November 2013. An abdominal drainage tube was placed in the pelvic cavity intraoperatively and a negative pressure suction device was positioned under the aponeurosis of the external oblique muscles postoperatively.

Results: The ascites of all patients were effectively controlled perioperatively. Surgery via the HST pathway was successful in all patients. The mean surgical duration was about $60 \mathrm{~min}$. There were no obvious postoperative complications, such as scrotum hematoma, seroma, bulging of the repaired area, severe pain, or hernia recurrence. This method also resulted in less scar formation, which helps to maintain tissue softness. All patients were satisfied with treatment.

Conclusion: Preperitoneal tension-free repair of inguinal hernia combined with ascites via the HST pathway was effective and maintained the integrity of the ventral transverse fascia. The HST pathway is a reliable choice for repair of inguinal hernia accompanied by ascites.

Mini-Abstract: Our purpose is to describe and evaluate the clinical value of preperitoneal tension-free repair of inguinal hernias combined with ascites via hernia-roof/hernia sac top (HST) pathway. We retrospectively reviewed the medical records of seven patients with inguinal hernia combined with ascites who underwent the new procedure. An abdominal drainage tube was placed in the pelvic cavity intraoperatively and a negative pressure suction device was positioned under the aponeurosis of the external oblique muscles postoperatively. Surgery was successful in all patients. All patients were satisfied with treatment and none experienced recurrence.

Keywords: Hernia sac top pathway; Inguinal hernia combined with ascites; Preperitoneal tension-free repair

\section{Zhong Jia*, Jie Zhang and Chao-Jun Kong}

Department of Hepatopancreatobiliary Surgery, Hangzhou First People's Hospital, Nanjing Medical, University Affiliated Hangzhou Hospital \& Zhejiang Traditional Chinese Medicine University Affiliated Training Hospital, China

Corresponding author: Jia Zhong

झ jiazhong20058@hotmail.com

Department of Hepatopancreatobiliary Surgery, Hangzhou First People's Hospital, Nanjing Medical University Affiliated Hangzhou Hospital \& Zhejiang Traditional Chinese Medicine University Affiliated Training Hospital, China.

Tel: +86-13958114181

Citation: Jia Z, Zhang J, Kong CJ (2017) Perioperative Management of Groin Ascitic Hernia by Hernia-roof Technique: A 14-year Experience. Arch Med. Vol. 9 No. 4:4

\section{Introduction}

Inguinal hernia combined with ascites is intractable and surgical repair is discouraged because of the risk of postoperative complications, such as hernia recurrence, bulging of the repaired area, and incision effusion due to continuous high abdominal pressure. In the past, we used a peritoneal dialysis catheter to control abdominal pressure perioperatively to ensure the safety and success of the surgery [1]. As an alternative, for the past 5 years, we adopted preperitoneal tension-free hernioplasty via the hernia sac top (HST) pathway with intraoperative placement of an abdominal drainage tube and a negative pressure suction 
device under the aponeurosis of the external oblique muscles to maintain the integrity of the ventral transverse fascia, which increases its ability to resist high abdominal pressure, while actively reducing the volume of ascites both intra- and postoperatively [2]. Here, we report our experience with preperitoneal tension-free repair via the HST pathway for repair of inguinal hernia combined with ascites.

\section{Patients and Methods}

From January 2010 to November 2013, seven males (average age, 62 years; age range, 32-65 years) underwent preperitoneal tension-free repair of an inguinal hernia combined with ascites via the HST pathway in the First People's Hospital (Hangzhou, China). All seven cases were intraoperatively diagnosed with indirect inguinal hernias due to posthepatitic cirrhosis in three cases and schistosomiasis cirrhosis, alcoholic cirrhosis, tumor combined with ascites, and unexplained ascites in one case each. Moreover, four cases had a moderate degree of ascites $(500 \mathrm{ml}$ or so), two had a moderate to large degree of ascites $(800 \mathrm{ml}$ or so), and one had a large degree of ascites (1200 $\mathrm{ml}$ or so). One case underwent emergency surgery for a strangulated inguinal hernia, while the others underwent elective surgery. Most of the hernia sac content and ascites were trapped small intestine. The maximum sac diameter was $10-15 \mathrm{~cm}$.

\section{Surgical Procedures}

\section{Preoperative treatment}

Many causes of inguinal hernia combined with ascites, including liver cirrhosis and tumor formation, may lead to decreased tolerance to treatment. Therefore, we initiated the following measures to ensure successful treatment: 1 . protect liver function; 2. diuresis to protect renal function and reduce the volume of ascites; 3. correct blood coagulation dysfunction; 4 . intravenous drip of antibiotics to prevent infection; 5 . correct electrolyte and acid-base imbalances; and 6 . correct hypoproteinemia.

\section{Hernioplasty via the HST pathway}

We used soft, light-weight, flat mesh for inguinal hernia repair via the HST pathway. All surgeries were performed under general anesthesia with the patients in the supine position. Through an incision of about $4 \mathrm{~cm}$, the skin, subcutaneous tissues, and external aponeurosis of the external oblique muscles are cut to expose the hernia sac. First, the hernia sac is located by opening the cremaster muscle and the tissues superior to the real hernia sac, which consists of the outer transversalis fascia. Second, the hernia sac is opened. If there is no necrosis, the content of the sac is returned to the abdominal cavity. Next, a small incision is made to the right or left lower quadrant to place an abdominal drainage tube in the pelvic cavity, which is then fixed in place. Afterward, the opening of the peritoneal anterior ring along the hernia sac, which consists of the deeper layer of the transversalis fascia, is located. This report is the first to describe hernia repair through this novel pathway. The size of the opening will be about $2-3 \mathrm{~cm}$. Through the opening, the preperitoneal space can be assessed immediately, which should be sufficient to place the flat mesh.
Then, after suturing the opened hernia sac, the preperitoneal space is separated and the mid zone of the flat mesh is fixed to the top of the hernia sac with absorbable sutures. Next, the leash is gripped with an oval clamp or forceps to push the flat mesh and hernia sac into the preperitoneal space and the mesh is fully flattened using the finger or forceps. Then, the opening of the transversalis fascia is closed. At the same time, the leash on the transversalis fascia is fixed and the extra leash is cut away. After dividing the cord, the upper mesh is placed behind the cord and conjoint tendon to enhance the firmness of the transverse fascia. Finally, after suturing, a negative pressure suction device is placed under the aponeurosis of the external oblique muscles

\section{Postoperative treatment}

The following actions were performed to ensure patient recovery, which can be summarized into three symptomatic treatment and two monitoring portions: 1 . The bowel is kept open with a drainage tube for one week to avoid high abdominal pressure, continue with routine therapy, facilitate supportive therapy, and prevent infection; 2 . Liver and kidney function, the fluid volume passing through the abdominal drainage tube, and negative pressure of the suction device are monitored. Usually after 3-5 days, when the fluid volume decreases to less than $10 \mathrm{ml} / \mathrm{day}$, the negative pressure suction device can be removed. Then, the abdominal drainage tube is removed 10-14 days later, while ensuring that the incision is fully healed.

\section{Results}

Inguinal hernia preperitoneal tension-free repair via the HST pathway was successful for all seven patients with sufficient healing of the incisions and no postoperative complications, such as scrotum hematoma, seroma, bulging of the repaired area, severe pain, or hernia recurrence. Above all, use of the HST pathway lessens scar formation, which is beneficial to maintain the softness of local tissue. All patients were satisfied with treatment and none experienced recurrence. The follow-up duration ranged from 2 years to 5 years. Only one of them died of heart stroke 2 years ago.

\section{Discussion}

Surgical repair of inguinal hernia combined with ascites is associated with several complications, such as incision effusion, hernia recurrence, and bulging of the repaired area, which is difficult to treat due to high abdominal pressure. Also, surgical repair of hernias with massive ascites, not only often fails, but can result in hepatic coma of patients with cirrhosis [3]. Thus, we previously used a peritoneal dialysis catheter to control abdominal pressure perioperatively to ensure the safety and success of the surgery. However, over the past 5 years, we often choose preperitoneal tension-free hernioplasty via the HST pathway and place an abdominal drainage tube and a negative pressure suction device under the aponeurosis of the external oblique muscles postoperatively to achieve the same goal of reducing pressure. Moreover, this method results in greater firmness of the repaired area than with other methods and can fully ensure the integrity of the ventral transverse fascia. 
Bassini et al. [4] first described inguinal hernioplasty in the 1880 s, which was followed by the Shouldice technique, which remains the gold standard for the prosthesis-free treatment of inguinal hernias. More recently, tension-free hernia repair has become increasingly popular, especially through the preperitoneal approach. However, reports of preperitoneal tension-free repair of hernias combined with ascites are relatively infrequent. We found that the anatomical structure of the ventral transverse fascia (preperitoneal loop) in open hernia repair was the same in the laparoscopic totally extraperitoneal approach [5], although this method receives little attention. We repair inguinal hernias via the HST pathway to maintain the integrity of the ventral transverse fascia, while conforming to the anatomy and the concept of tension-free more to ensure good patient recovery and comfort. In this method, the distal hernia sac is returned under the outer layer of the ventral transverse fascia to avoid postoperative scrotum hematoma formation and reduce the incidence of hernia recurrence [6-13].

\section{References}

1 Jia Z, He GH (2005) Inguinal hernioplasty in patients with liver cirrhosis accompanied by ascites. Bull Med Res 34: 23-24.

2 Chen J, LiuSJ (2009) Umbilical hernia repair in patient with ascites. Chin J Gen Surg 24: 878-881.

$3 \mathrm{Hu} \mathrm{Q}, \mathrm{Ma} \mathrm{Y}$ (2001) Perioperative treatment in patients with abdominal external hernia accompanied by cirrhosis. Natl Med J China 81: 371-372.

4 Amato B, Moja L, Panico S, Persico G, Rispoli C, et al. (2012) Shouldice technique versus other open techniques for inguinal hernia repair. Cochrane Database Syst Rev 18: 4.

5 Cavazzola LT, Rosen MJ (2013) Laparoscopic versus open inguinal hernia repair. Surg Clin North 93: 69-79.

6 Gilbert Al, Graham MF (2001) Tension-free hernioplasty using a bilayer prosthesis[M]//Fitzgibbons RJ Jr,Greenburg AG. Nyhus and Condon's hernia. [5 Edn] Philadelphia: Lippincott William \& Willkins, 173-180.

7 Berger D (2014) Diagnostics and therapy of chronic pain following hernia operation. Chirurg 85: 117-120.

\section{Conclusion}

Groin hernia repair via the HST pathway is a safe and simple method, although there are no domestic or overseas reports of this procedure in the literature. Nonetheless, our proposed method conforms to human physiology and anatomy, and is beneficial to maintain the integrity of the ventral transverse fascia and the preperitoneal loop. Moreover, this approach is easy to learn with a reliable outcome and can be applied for repair of hernias combined with ascites.

\section{Acknowledgement}

The study was sponsored by Hangzhou Science and Technology Program of Zhejiang Province (No.20150733004)

\section{Conflict of Interest}

The authors declare no conflicts of interest associated with this report.

8 Hassan AM, Salama AF (2014) Outcome of sublay mesh repair in non-complicated umbilical hernia with liver cirrhosis and ascites. Int J Surg 12: 181-185.

9 Peitsch WK (2013) A modified laparoscopic hernioplasty (TAPP) is the standard procedure for inguinal and femoral hernias: A retrospective 17-year analysis with 1,123 hernia repairs. Surg Endosc 28: 671-682.

10 Williams KB, Bradley JF, Wormer BA, Zemlyak AY, Walters AL, et al. (2013) Postoperative quality of life after open transinguinal preperitoneal inguinal hernia repair using memory ring or threedimensional devices. Am Surg. 79: 786-793.

11 Gillion JF, Chollet JM (2013) Chronic pain and quality of life (QoL) after transinguinalpreperitoneal (TIPP) inguinal hernia repair using a totally extraperitoneal, parietalized, Polysoft memory ring patch: A series of 622 hernia repairs in 525 patients. Hernia.

12 Cavazzola LT, Rosen MJ (2013) Laparoscopic versus open inguinal hernia repair. Surg Clin North Am 93:1269-1279.

13 Odom SR, Gupta A (2013) Emergency hernia repair in cirrhotic patients with ascites. J Trauma Acute Care Surg 75: 404-409. 